\title{
Une collecte de littérature orale chez les Dioula de Kong (Côte d'ivoire) : témoignage
}

Jean Derive

\section{(2) OpenEdition}

1 Journals

Édition électronique

URL : https://journals.openedition.org/clo/197

DOI : $10.4000 /$ clo. 197

ISSN : 2266-1816

Éditeur

INALCO

\section{Édition imprimée}

Date de publication : 1 janvier 2008

Pagination : 171-183

ISBN : 978-2-85831-181-1

ISSN : 0396-891X

Référence électronique

Jean Derive, "Une collecte de littérature orale chez les Dioula de Kong (Côte d'ivoire) : témoignage », Cahiers de littérature orale [En ligne], 63-64 | 2008, mis en ligne le 29 décembre 2011, consulté le 07 juillet 2021. URL : http://journals.openedition.org/clo/197 ; DOI : https://doi.org/10.4000/clo.197

Ce document a été généré automatiquement le 7 juillet 2021.

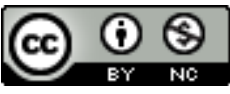

Cahiers de littérature orale est mis à disposition selon les termes de la Licence Creative Commons Attribution - Pas d'Utilisation Commerciale 4.0 International. 


\title{
Une collecte de littérature orale chez les Dioula de Kong (Côte d'ivoire) : témoignage
}

\author{
Jean Derive
}

1 La question de la collecte des genres oraux dans une communauté est toujours une question particulièrement délicate. Les modalités de cette opération sont en effet conditionnées :

- d'une part, par les modalités habituelles de production et de consommation de cette littérature orale, qui n'existent parfois plus que sous forme de traces archaïques qu'il convient d'activer;

- d'autre part, par les visées du collecteur qui déterminent à la fois :

1. la nature de l'objet ou des objets qu'il veut recueillir (un genre, plusieurs genres, des répertoires de genres en fonction du statut social des interprètes, une œuvre - en une ou plusieurs versions -, etc.) ;

2. les conditions dans lesquelles il doit les recueillir (exigence qu'elles soient plus ou moins naturelles selon qu'il s'intéresse seulement au contenu ou à la stylistique de l'oralité et à l'art de la performance).

2 Par ailleurs, la problématique du terrain se pose en des termes très différents selon le statut de l'enquêteur. Elle n'est pas du tout la même selon qu'il enquête dans une communauté appartenant à sa propre culture, où il est éventuellement connu depuis sa tendre enfance, dans laquelle il peut avoir de la famille ou des amis, à propos de laquelle il dispose a priori d'un très grand nombre d'informations acquises par imprégnation naturelle, ou selon au contraire qu'il est étranger à cette culture dont il ne connaît qu'imparfaitement la langue et sur laquelle il a beaucoup à apprendre, même si des lectures préliminaires l'ont parfois préparé à son accès.

Cette problématique est aussi déterminée par les conditions selon lesquelles le chercheur a la possibilité d'effectuer ses séjours de terrain, suivant qu'ils sont fréquents ou pas, qu'ils sont brefs (quelques jours, quelques semaines) ou longs (parfois plusieurs années en continu, comme dans le cas de beaucoup de missionnaires religieux 
qui s'intéressent au folklore des communautés où ils exercent leur ministère). Il est évident en effet que plus le séjour est long, plus il est possible de suivre le cours naturel des choses, de recueillir les données au fur et à mesure qu'elles se présentent, sans rien forcer, ce qui est toujours préférable.

4 Le témoignage qui va suivre concerne une campagne de collecte déjà ancienne puisqu'elle s'est déroulée de 1975 à 1982 dans la sous-préfecture de Kong, au nord-est de la Côte d'Ivoire, ancienne capitale d'un royaume dioula fondé par Sekou Watara (Ouattara, selon l'usage administratif) au début du xviII siècle. C'est cette agglomération, alors peuplée de deux mille six cents habitants et toujours composée essentiellement de Dioula qui fut retenue comme un ensemble social cohérent de production et de consommation de littérature orale. L'enquête de terrain, dans tous ses aspects, était orchestrée par moi-même, à savoir un enseignant-chercheur français, étranger à la culture approchée dont la connaissance n'était au départ que livresque et partant fort partielle.

5 Pour comprendre les conditions suivant lesquelles cette campagne fut menée, il faut commencer par exposer les finalités du projet dont elle constituait l'étape préliminaire. Il s'agissait :

- d'une part de recueillir le maximum de genres du discours reconnus par un nom spécifique dans la taxonomie locale et dont l'énoncé comme l'énonciation obéissaient à un certain nombre de règles canoniques ;

- d'autre part de considérer cet ensemble codifié comme un système, chaque genre définissant ses fonctions socioculturelles par rapport aux autres, d'après à la fois le contenu et le style des énoncés produits dans ce cadre canonique, données corrélées aux ensembles de qui peut (ou doit) le dire et de qui peut (ou doit) l'entendre;

- en dernier lieu, il s'agissait aussi de déterminer si, dans les usages parlés des Dioula de Kong, il existait des expressions consacrées et plus ou moins récurrentes, susceptibles de tracer des frontières entre différents aspects de leur activité verbale et si, dans l'hypothèse d'une réponse positive, les distinctions ainsi opérées, permettaient d'isoler un champ de l'activité discursive ayant un certain nombre de traits plus ou moins comparables à ce qu'on nomme « littérature » dans les sociétés de l'écriture.

6 Ces trois objectifs n'engageaient pas tout à fait des opérations de même nature puisque l'un consistait surtout à faire de la collecte d'œuvres, alors que les deux autres appelaient davantage des enquêtes de terrain auprès des intéressés. Pour mener à bien ces opérations, un minimum de connaissances linguistiques était nécessaire. Comme je n'avais pas eu l'occasion d'étudier le dioula auparavant, j'ai choisi de faire préalablement un stage en cette langue, proposé à l'époque par l'université d'Abidjan, afin d'acquérir quelques bases.

7 Ces premières connaissances, plutôt rudimentaires, ne permettaient certes ni de comprendre d'emblée les œuvres enregistrées (notamment les plus difficiles) ni de mener seul des enquêtes auprès de la population, sans le recours d'un informateurinterprète. Elles offraient néanmoins la possibilité, grâce en particulier à une connaissance phonologique, de pouvoir mieux former des transcripteurs selon les normes de la graphie officielle, de mieux suivre leur travail et d'avoir quelque prise sur le mot à mot des textes dans les premières entreprises de traduction. Par ailleurs, elles permettaient de conserver un minimum de contrôle sur les enquêtes, en vérifiant que les interventions de l'enquêteur autochtone respectaient l'esprit des objectifs fixés. 
8 En outre, dans la mesure où la campagne de terrain a duré sept ans et qu'elle a occasionné des séjours sur place assez longs (entre deux semaines et un mois) et assez fréquents (j'enseignais alors à l'université d'Abidjan), il m'a été possible de progresser assez rapidement dans la pratique de la langue, en conversant quotidiennement en dioula avec les habitants de Kong et en entretenant ces acquis linguistiques à Abidjan, le dioula étant aussi, sous une forme légèrement différente, une langue véhiculaire de Côte d'Ivoire ; au point qu'à la fin de la campagne, il m'est arrivé encore assez souvent de mener les enquêtes moi-même, l'informateur n'étant là que pour intervenir ponctuellement en cas de difficulté de communication.

9 Les trois objectifs évoqués étaient interdépendants et par conséquent ne représentaient pas trois étapes successives distinctes d'opérations à conduire, la seconde ne pouvant être entamée que lorsque la première était terminée et ainsi de suite. Ces opérations ont donc été menées de front dès le début du travail de terrain.

10 C'est ainsi que la collecte de performances orales plus ou moins ritualisées a commencé très vite, dès le premier séjour, à l'occasion de séances de contes et de devinettes qui s'étaient organisées spontanément dans certains quartiers de Kong et dont j'avais eu vent grâce aux premiers contacts pris avec la population, notamment les jeunes scolarisés. Ces premiers enregistrements au magnétophone furent très tôt suivis par la collecte de chants cérémoniels exécutés à l'occasion de mariages, de baptêmes, de circoncision, de sorties de masques, de retours de pèlerinage à La Mecque, de fêtes calendaires, etc. Dans une agglomération de deux mille six cents habitants, il est rare qu'un séjour de quelques semaines n'offre pas l'opportunité d'assister à l'une ou à l'autre de telles performances. Et, lorsqu'on a effectué, sur plusieurs années, une vingtaine de séjours, situés à des périodes différentes du calendrier, l'occasion est offerte de découvrir une palette de genres canoniques qui est loin d'être négligeable.

11 Il convenait toutefois, pour toutes ces séances, de demander aux intéressés la permission d'assister et d'enregistrer, ce qui, en l'occurrence, ne fut la plupart du temps qu'une formalité de courtoisie, l'accord étant donné bien volontiers. Une exception peut être faite avec certains chants de masques qui sont réservés aux seuls initiés. Pour me permettre d'être présent lors de ces performances à l'auditoire restreint, on me fit subir un simulacre d'initiation (afin surtout de sauvegarder les apparences aux yeux de la population) et on me fit promettre de ne pas enregistrer ces chants et de n'en rien diffuser auprès de quiconque n'appartenait pas au cercle des initiés, condition que j'ai bien entendu respectée.

12 J'avais opté en effet pour le parti de commencer au moins la campagne de collecte par des performances produites «naturellement» par la communauté en dehors de toute sollicitation de ma part. Cette condition était nécessaire dans la mesure où au moins deux objectifs de l'étude consistaient :

- d'une part à observer qui produisait et qui consommait quels discours ;

- d'autre part à étudier le style de la performance dans tous ses paramètres (gestuelle, kinésique, interactivité entre le [les] interprète[s] et son [leur] auditoire).

13 De telles préoccupations ne pouvaient être satisfaites que si les genres du discours en question étaient produits aux occasions habituelles devant leur public naturel, en dehors de toute intervention artificielle. 
Pour pouvoir être exploitable aux fins de l'étude projetée, la collecte de chaque performance devait être assortie d'un certain nombre d'informations relatives aux conditions d'exécution, consignées sur un cahier :

- temps de l'exécution (moment de la journée aussi bien que date calendaire);

- lieu de l'exécution (intérieur d'une maison, cour familiale, place publique d'un quartier, itinéraire à l'intérieur d'un ou de plusieurs quartiers (cas notamment de certains chants de masques ou de certains chants nuptiaux) ;

- accompagnement musical éventuel (cas de la grande majorité des genres oraux de Kong): nature des instruments, nature des musiciens... Des enquêtes a posteriori, de même que d'autres enregistrements de la même catégorie d'œuvres, pouvaient aider à déterminer si ces musiciens étaient toujours les mêmes, nominalement parlant, ou s'ils étaient interchangeables à l'intérieur d'une catégorie sociale donnée ;

- nombre (précis dans le cas de petits groupes, approximatif dans le cas d'une foule) et nature sociale des partenaires concernés : principalement âge, sexe, appartenance à un quartier, à une caste, éventuellement à une fonction, etc., la pertinence des critères étant à évaluer pour chaque cas particulier (par exemple, dans le cas des chants nuptiaux de Kong, une distinction tout à fait pertinente était à faire entre les femmes mariées et les jeunes filles non encore mariées, mais en âge de l'être, les premières et les secondes ayant un répertoire de chants différent). Il va de soi que, lorsque l'énonciation n'était pas entièrement collective (il faut entendre par énonciation collective le cas où certains chants sont produits en chœur par la totalité de l'assemblée), parmi ces partenaires de la performance, étaient à distinguer d'une part les énonciateurs, d'autre part les récepteurs du discours, les uns et les autres n'ayant pas forcément le même statut social. Il est des cas où tous les membres présents sont des énonciateurs potentiels et d'autres où, en revanche, dans l'ensemble des partenaires présents, il y a des énonciateurs prescrits et des consommateurs prescrits qui appartiennent à des groupes sociaux distincts. Pour ce qui est des interprètes, la précision pouvait aller jusqu'à les dénommer nominalement. Cette précaution offrait l'avantage d'affiner au mieux la connaissance du système d'appropriation des genres et, dans la mesure où les interprètes étaient bien identifiés, de pouvoir approfondir certains points auprès d'eux après coup ;

- relevé de certains traits propres à la performance orale: kinésique et proxémique (déplacements éventuels des énonciateurs et de l'auditoire, danse, gestuelle...). Quelques enregistrements de certaines des performances les plus remarquables, filmés au magnétoscope, ont permis d'avoir un aperçu de ces données in vivo et de pouvoir les exploiter après collecte.

15 Quand un nombre suffisant d'œuvres appartenant à un genre donné avait été enregistré de façon naturelle, il devenait possible d'en recueillir éventuellement d'autres versions dont l'énonciation avait été provoquée, encore que l'idéal reste l'exécution spontanée. Mais il est certains genres qui n'ont l'occasion de se produire que rarement ou encore de façon très aléatoire et il serait dommage de se priver de la richesse d'un répertoire au nom du principe rigide de la collecte naturelle. Ainsi, les proverbes par exemple, qui se disent dans la conversation courante, sont encore assez difficiles à traquer, car il est presque impossible de prévoir leur apparition et donc d'anticiper leur enregistrement. C'est pourquoi à un échantillon recueilli en situation naturelle au hasard des conversations avec les informateurs, en a été ajouté un autre qui était le résultat d'une enquête orientée vers ce genre parémique.

16 Les enregistrements en présence du chercheur, qui sait mieux que quiconque les données dont il a besoin, sont évidemment l'idéal et je me suis toujours efforcé, à 
l'occasion de mes séjours à Kong, d'être très vigilant, afin de ne laisser échapper que le moins possible de ce qui se produisait dans tel ou tel quartier (Kong en comprend neuf) en matière de production verbale canonique. Cela suppose de mettre en place un réseau d'informateurs fiables et rémunérés dans les différents quartiers de l'agglomération, car il n'est évidemment pas possible d'être intime avec chaque famille.

Mais, malgré tout, une présence effective limitée à deux mois environ par année, même si elle se répète sur plusieurs années, laisse échapper beaucoup de productions et risque notamment de ne pas permettre la collecte de certains genres saisonniers, voire même exécutés à certaines dates précises.

Pour pallier cet inconvénient, dès la deuxième année d'enquête, $\mathrm{j}$ 'ai formé un groupe de collecteurs (un dans chaque quartier de Kong, soit neuf en tout), choisis parmi les jeunes garçons ayant reçu une scolarisation au niveau du primaire (les adolescentes n'étaient guère scolarisées), c'est-à-dire ayant un niveau de français acceptable à l'oral comme à l'écrit. Grâce aux crédits du LACITO $^{1}$ que j'avais obtenus pour cette recherche et au soutien dont je bénéficiais de la part de plusieurs instituts de l'Université d'Abidjan (ILA, ILENA, GRTO'), ces collecteurs adjoints ont pu recevoir chacun une petite rémunération et être dotés d'un mini-magnétophone assorti d'un lot de cassettes. En échange, ils devaient enregistrer les performances culturelles verbales qui advenaient dans leur quartier lorsqu'ils en avaient connaissance, sous réserve évidemment de l'accord des intéressés. Ils disposaient également pour cela d'un cahier dans lequel leur étaient indiquées toutes les informations contextuelles à recueillir conjointement à chaque exécution (les mêmes que celles que j'ai déjà indiquées sur le temps, le lieu, les partenaires et autres circonstances de la performance). Ils devaient d'abord donner ces informations oralement sur la bande du magnétophone, juste avant le début de la séance, et les retranscrire ensuite sur leur cahier.

La mise en place d'une telle procédure m'a permis de doubler la collecte in praesentia et de recueillir plusieurs genres de discours dont je n'aurais peut-être jamais soupçonné l'existence. Cela m'a également donné l'occasion d'avoir une gamme plus étendue d'œuvres dans le répertoire d'un même genre ainsi qu'un échantillon plus riche de variantes d'une même œuvre, ce qui éclaire beaucoup le processus de création en oralité, entre reproduction d'un répertoire mémorisé et actualisation personnelle, dans le respect de règles canoniques, en fonction de certains contextes. Enfin, grâce à cette collecte aux maillons plus serrés, j'ai pu avoir une idée beaucoup plus précise de l'étiquette de l'appropriation de la parole par différents groupes sociaux.

Cette collecte, au fur et à mesure qu'elle s'opérait, s'accompagnait d'enquêtes parallèles qui, quant à elles, ont toujours été faites en ma présence et sous mon contrôle. La plupart des enregistrements étaient réécoutés par différents membres de l'auditoire, choisis de sorte qu'on ait un échantillon social diversifié (par exemple homme/femme, vieux/jeune, noble/captif, etc.) si du moins cet auditoire n'était pas socialement homogène, ce qui est la plupart du temps le cas. En effet, si certains genres ne concernent par exemple que les enfants, ou les adolescentes, ou les vieilles femmes, il est vrai qu'au sein du groupe, on trouvera encore des distinctions de classes (nobles/ captifs, scolarisés/non scolarisés...). Ces informateurs étaient invités à discourir sur la performance à laquelle ils avaient assisté et qu'ils réécoutaient sur le magnétophone, pour exprimer leur satisfaction ou leurs réserves.

21 Parallèlement, d'autres enquêtes étaient menées auprès d'informateurs qui n'avaient pas assisté à la performance en question. Cela permettait d'avoir une idée plus précise 
de l'aire d'extension du genre considéré et d'évaluer jusqu'à quel degré il était connu et apprécié à l'intérieur de la communauté dans d'autres sphères que celle des producteurs et consommateurs. Dans certains cas, nous avons pris le parti de porter simplement à la connaissance de ces informateurs le contenu linguistique de l'énoncé, sans leur faire écouter l'enregistrement. Cette stratégie nous a permis de constater l'embarras fréquent de certains informateurs pour déterminer à quel genre appartenait l'œuvre qui leur était soumise en l'absence de certaines informations : qui l'avait dit, où, quand, sous quelle forme (parlée, chantée, avec ou sans accompagnement musical, etc.) ? Ce type d'expérimentation nous a permis progressivement de comprendre qu'en oralité, contrairement à ce qui se passe dans la culture de l'écriture, souvent un genre se définissait prioritairement par les paramètres de son énonciation et subsidiairement seulement par les paramètres de son énoncé.

Toutes les enquêtes menées auprès de la population l'étaient de façon aussi peu directive que possible. Il est bien connu en effet que l'enquête directive est extrêmement périlleuse, car elle expose à tous les écueils de l'ethnocentrisme, surtout si l'enquêteur ne provient pas du même horizon culturel. Qu'il le veuille ou non, celui-ci part de ses catégories mentales pour essayer de comprendre les modes de représentation de l'Autre. Dans le cadre d'une enquête directive, ce dernier est donc soumis à un cadre préétabli qui lui est étranger et dont, pour cause, il ne saisit pas le plus souvent la logique, pas plus que l'enquêteur n'est lui-même préparé à celle qui régit le système de représentation de celui qu'il interroge. Une telle situation favorise les quiproquos et autres ratés de la communication interculturelle. Cela est d'autant plus vrai, comme l'ont mis au jour plusieurs exemples d'enquêtes ethnologiques, que la personne qui fait l'objet de l'enquête, par courtoisie, par intérêt ou simplement par lassitude, cherche à complaire à celui qui la questionne et peut, par bonne volonté, acquiescer à des questions inductives, réponses qui ne correspondent en rien à la réalité de ses pratiques ou de ses représentations.

Il est donc préférable de ne pas enfermer l'informateur dans un réseau serré de questions préparées à l'avance, mais de le laisser discourir librement, à partir de quelques sollicitations aussi vagues et générales que possible, en le laissant avancer à son gré et en ne le relançant que sur des propos qu'il a lui-même tenus pour avoir des précisions ou des éclaircissements ; cela même si son discours paraît au premier abord déroutant, voire sans intérêt par rapport à ce qu'on cherche. Si ces témoignages sont enregistrés, ce que nous avons fait systématiquement, c'est dans l'analyse a posteriori de cette série de métadiscours que le chercheur peut essayer de repérer des récurrences susceptibles d'être l'indice de représentations culturelles et collectivement partagées, au-delà des subjectivités individuelles.

Parmi ces récurrences, il convient de porter notamment son attention sur des expressions qui reviennent assez systématiquement d'une enquête à l'autre et qui ont par conséquent de bonnes chances de correspondre à des concepts communautaires portés par des idiotismes figés. Ce sont ces noyaux de représentation culturelle, dont la portée sémantique s'enrichit et se précise par les différents contextes où ils sont employés dans les gloses, qui peuvent, s'ils sont avérés comme concepts collectifs, servir de base à la mise au jour d'un système de représentation de l'exercice du discours au sein de la société.

Il convient toutefois d'être prudent, car le risque existe pour l'enquêteur de confondre une simple périphrase, création subjective de telle ou telle personne interrogée, avec 
une expression idiomatique consacrée par l'usage. Pour s'assurer de l'existence d'un véritable idiotisme porteur d'un concept, il faut donc d'une part, s'appuyer à la fois sur une récurrence interindividuelle importante, d'autre part s'assurer ensuite que l'expression relevée a bien un écho auprès d'un échantillon significatif de la population.

Cette ligne de conduite que j'ai suivie au cours de la campagne a ainsi permis de faire émerger, dans la pratique discursive des Dioula, un certain nombre d'expressions consacrées à valeur hyperonymique, notamment à propos de la catégorisation des discours patrimoniaux dont la mémoire était conservée dans des répertoires.

C'est ainsi, à un premier niveau, que j'ai pu découvrir qu'un certain nombre de genres que j'avais enregistrés et dont j'avais découvert le nom spécifique à l'occasion de leur exécution, étaient susceptibles de s'intégrer dans des classes plus larges fonctionnant comme des catégories génériques. Ainsi en allait-il des kònyon dònkili (chants de mariage), des dòn dònkili (chants de danse), des dò dònkili (chants de masques), catégories englobant chacune une dizaine de genres spécifiques ayant leur dénomination propre. Par exemple, les fanila dònkili (chants du pagne) ou les kúrubi dònkili (chants de déploration)... exécutés dans le cadre des cérémonies nuptiales, font partie de la catégorie des chants de mariage ; les kúrubi dònkili (chants donnés à la fin du ramadan) et les màdeun dònkili (chantés au retour de pèlerinage) appartiennent, avec beaucoup d'autres, à la catégorie des chants de danse ; les dòndo dònkili (chants du coq), les gbòn dònkili (chants du cynocéphale) relèvent quant à eux de la catégorie des chants de masques.

À un niveau sémantique plus large, les métadiscours autochtones ont fait apparaître d'autres expressions plus générales pour classer l'activité discursive. En premier lieu, j'ai pu relever, dans l'analyse de ces gloses deux expressions qui revenaient systématiquement et qui fonctionnaient comme une paire à valeur discriminante, les discours produits par la communauté appartenant nécessairement soit à l'une soit à l'autre de ces catégories. Il s'agissait des expressions suivantes: kúma gbé (parole ${ }^{3}$ claire, au sens où on l'entendrait en français, c'est-à-dire parole facilement compréhensible) et kúma kòro (parole vieille, c'est-à-dire ancienne ou, en d'autres termes, issue d'une tradition).

On remarque tout de suite que cette paire discriminante n'est pas une paire antonymique, sémantiquement parlant. Cette apparente absence de logique permet en fait d'enrichir l'opposition entre les deux expressions. Puisqu'elles sont discriminantes, on comprend donc que la parole qui n'est pas ancienne (comprendre pas authentifiée par un patrimoine) est claire (immédiatement accessible), tandis que la parole relevant, elle, d'une tradition patrimoniale, n'est pas claire (comprendre qu'elle n'est pas accessible avec la seule maîtrise du code linguistique, le discours se trouvant surcodé par d'autres systèmes symboliques et poétiques, qu'il faut connaître pour accéder au sens). Il est donc progressivement apparu qu'il existait, chez les Dioula, la conscience nette d'une distinction entre les discours ordinaires et les discours de tradition et que la représentation culturelle consacrée prêtait à chacun d'eux des propriétés sinon réelles du moins idéologiques. Les discours ordinaires étaient réputés accessibles (non ésotériques), les discours de tradition au contraire étaient réputés ne livrer leur sens qu'après un décorticage de métaphores et de références contextuelles accessibles aux seuls initiés, c'est-à-dire aux gens de culture.

30 Cette hypothèse s'est trouvée confortée avec l'apparition, dans les commentaires des informateurs, d'une autre expression qui s'appliquait sans cesse aux kúma kòro. Ces 
paroles anciennes étaient toujours qualifiées de kòro kúma, le substantif kòro (à ton haut cette fois) désignant le fond (invisible) d'un récipient lorsqu'il repose sur le sol, mais aussi le sens caché d'un énoncé. Il s'agit donc de discours dont le fond caché (i.e. la signification profonde) est à découvrir, au-delà du signifié immédiat. L'équation kúma kòro = kòro kúma a ainsi progressivement émergé des commentaires.

Une distinction de second degré a pu également être repérée, grâce à sa récurrence, à l'intérieur de la classe des discours de tradition. Un grand nombre de nos informateurs avait tendance à opposer systématiquement dans cet ensemble deux catégories de paroles qu'ils appelaient tólon kúma d'une part, et kúmaba d'autre part.

La première expression signifie littéralement "parole de jeu " ou encore "parole de plaisanterie». J'ai progressivement compris, grâce à la diversité des contextes d'emploi, que malgré son intitulé, cette classe de discours canoniques ne s'appliquait pas seulement aux comptines des enfants ni aux blagues et autres histoires drôles comme on en trouve dans toutes les cultures. Elle servait en fait à désigner tout type de discours dont l'idéologie dominante disait qu'il n'était pas à prendre au sérieux : ainsi étaient, entre beaucoup d'autres, les contes, les devinettes, beaucoup de chants de jeunes filles ou de joutes oratoires entre adolescents.

33 La deuxième expression, quant à elle, qui voit le suffixe augmentatif et mélioratif «ba » s'accoler au mot kúma (discours) et qu'on peut par conséquent traduire par quelque chose comme "discours important", s'appliquait à toutes les formes de discours patrimoniaux qui étaient censés avoir du poids. Dans cette catégorie se trouvaient, parmi bien d'autres, les énoncés sentencieux, les récits étiologiques, les récits épiques, les chroniques historiques, les devises familiales...

L'attestation de telles formulations comme expressions consacrées, corrélées à un certain nombre d'autres observations, notamment sur les règles d'appropriation de la parole en fonction du statut social, a permis d'ébaucher les grandes lignes de la représentation consensuelle (celle de l'idéologie dominante en tout cas) du système de fonctionnement de la parole chez les Dioula de Kong ${ }^{4}$. On peut, en gros décrire ce système comme suit : les Dioula opposent consensuellement un discours ordinaire et un discours de tradition, comme en témoigne l'expression kúma kòro (discours ancien). Cette dénomination n'implique pas forcément que le discours en question s'applique nécessairement à une œuvre déjà constituée et consignée comme telle dans un répertoire. Elle concerne également tout discours ritualisé, susceptible d'être rattaché à un genre connu et nommé, élaboré selon un certain nombre de règles canoniques admises.

Dans le champ ainsi délimité des discours de tradition, que par analogie on pourrait considérer comme relevant d'une sorte de « littérature » orale (puisqu'ils s'élaborent canoniquement en fonction d'une poétique connue), les Dioula opposent des paroles sans conséquence (tólon kúma) à des paroles de poids (kúmaba). Les observations relevées à propos des paroles de la première catégorie (grâce aux informations complémentaires collectées, complétées par les enquêtes) montrent qu'elles ne sont guère contrôlées par une étiquette (elles peuvent le plus souvent être proférées à n'importe quelle occasion et par n'importe qui) ou bien que, si elles sont l'apanage d'une catégorie sociale déterminée, elles sont le fait des jeunes, des femmes, des captifs, c'est-à-dire de formations sociales dominées ou dotées de peu de crédit. Ce sont des paroles ludiques ou, en tout cas, cathartiques. Les genres appartenant à la deuxième catégorie, au contraire, voient leur énonciation très étroitement contrôlée par un 
protocole et sont aux mains de formations sociales valorisées ou de groupes assujettis à elles (griots) : hommes d'âge, chefs temporels ou religieux... Les discours produits dans ce cadre sont d'abord des discours de légitimation.

Ce simple témoignage d'une expérience de terrain, qui a connu ses tâtonnements et ses errements (je me souviens très bien de mes angoisses devant mon incompréhension à propos de certains commentaires d'informateurs ainsi que des premières hypothèses sur quelques concepts autochtones qui se sont révélées erronées par la suite) n'est donc pas un modèle à suivre absolument pour tout terrain de collecte et d'enquête en matière de littérature orale. L'exposé succinct de cette expérimentation a simplement voulu mettre en évidence l'importance capitale de la corrélation entre les informations à recueillir et les ambitions analytiques de l'étude. Ces analyses ne peuvent en effet se faire de façon valable que d'après certaines données qui doivent avoir été établies à l'occasion de la collecte et des enquêtes qui l'accompagnent, en fonction des besoins du chercheur. C'est pourquoi une campagne de terrain ne peut démarrer de façon efficace que si celui-ci a au départ une idée de ce qu'il veut chercher. En même temps, il est vrai que des découvertes inattendues, offertes par les hasards du terrain, peuvent infléchir le projet initial et donner une nouvelle direction, non prévue, à l'étude. Il y a là tout un processus dialectique auquel il convient d'être sensible si l'on veut faire une recherche dynamique.

\section{BIBLIOGRAPHIE}

DERIVE, Jean, 1987a, Le fonctionnement sociologique de la littérature orale. L'exemple des Dioula de Kong (Côte d'Ivoire), Paris, Institut d'Ethnologie, coll. « Sciences Humaines », Archives et documents, $2326 \mathrm{p}$.

DeRIVE, Jean, 1987b, Parole et pouvoir chez les Dioula de Kong, Journal des Africanistes, 57, « Les voix de la parole », p. 19-30.

DERIVE, Jean, 1989, Le Jeune menteur et le vieux sage, Graines de parole (Écrits pour Geneviève Calame-Griaule), Paris, Éditions du CNRS, p. 185-200.

\section{NOTES}

1. UMR CNRS : LAngues et CIvilisations de Tradition Orale (LACITO).

2. Institut de Linguistique Appliquée, Institut de Littérature et d'Esthétique Négro-Africaine, Groupe de Recherche en Tradition Orale.

3. Le mot kúma désigne à la fois la parole et le discours.

4. Pour plus de détail sur ce système, on pourra consulter notamment Jean Derive (1987a, 1987b, 1989). 


\section{RÉSUMÉS}

Ainsi que l'indique le titre, cette contribution entend d'abord témoigner, hors de tout dogmatisme, à propos d'une expérience de collecte qui n'est certes pas un modèle, mais qui présente les stratégies adoptées pour répondre au mieux à certains choix politiques dictés par les objectifs d'une étude visant à étudier le système culturel formé par l'ensemble des genres oraux d'une communauté et par conséquent à disposer de données propres à analyser leurs conditions de production et de consommation. Ces objectifs impliquaient au moins deux exigences: rechercher l'exhaustivité, sinon en matière d'œuvres (ce qui est impossible avec les répertoires patrimoniaux de l'oralité), du moins en matière de genres, prétention qui suppose une présence continue sur le terrain pendant plusieurs cycles de saisons ; recueillir dans la mesure du possible les œuvres orales en situation naturelle, c'est-à-dire lorsqu'elles se produisent normalement sans l'intervention extérieure d'enquêteurs. Comme la plupart du temps, il n'est pas possible au chercheur de rester de façon permanente sur le terrain, l'article présente, à partir d'une expérience personnelle, quelques solutions possibles pour mettre en place des relais fiables, susceptibles non seulement de permettre, in abstentia, la collecte des œuvres orales, lorsqu'elles viennent à se produire, mais aussi celle des informations contextuelles qui seront ensuite nécessaires à l'analyse.

This article describes a fieldwork experience which, while it may not be model, adopted certain strategies in order to conduct an exhaustive study of the different genres in a given society and to collect oral texts in their performance context - as they are produced naturally without the intervention of an outsider. Since most of the time, it is not possible to stay permanently in the field, the outside researcher must put in place a system of relays who can collect in context and in the scholar's absence and who can also provide the necessary contextual information that will be necessary for analysis.

\section{INDEX}

Thèmes : anthropologie (Afrique)

Mots-clés : enquête collective, expérimentation, genres oraux, système culturel

nomsmotscles Dioula

Index géographique : Kong, Côte d'Ivoire

Keywords : Collective Investigation, Experiment, Dioula (Ivory Coast), Oral Genres, Cultural System, Anthropology 